\title{
Maternal Fructose Intake Induces Insulin Resistance and Oxidative Stress in Male, but Not Female, Offspring
}

\author{
Lourdes Rodríguez, ${ }^{1}$ Paola Otero, ${ }^{1}$ María I. Panadero, ${ }^{1}$ Silvia Rodrigo,, \\ Juan J. Álvarez-Millán, ${ }^{1,2}$ and Carlos Bocos ${ }^{1}$ \\ ${ }^{1}$ Facultad de Farmacia, Universidad CEU San Pablo, Urbanización Montepríncipe, Boadilla del Monte, 28668 Madrid, Spain \\ ${ }^{2}$ CQS Laboratory, C/Artistas 1, 28020 Madrid, Spain \\ Correspondence should be addressed to Carlos Bocos; carbocos@ceu.es
}

Received 15 October 2014; Accepted 14 January 2015

Academic Editor: Phillip B. Hylemon

Copyright (C) 2015 Lourdes Rodríguez et al. This is an open access article distributed under the Creative Commons Attribution License, which permits unrestricted use, distribution, and reproduction in any medium, provided the original work is properly cited.

Objective. Fructose intake from added sugars correlates with the epidemic rise in metabolic syndrome and cardiovascular diseases. However, consumption of beverages containing fructose is allowed during gestation. Recently, we found that an intake of fructose $(10 \% \mathrm{wt} / \mathrm{vol})$ throughout gestation produces an impaired fetal leptin signalling. Therefore, we have investigated whether maternal fructose intake produces subsequent changes in their progeny. Methods. Blood samples from fed and $24 \mathrm{~h}$ fasted female and male 90 day-old rats born from fructose-fed, glucose-fed, or control mothers were used. Results. After fasting, HOMA-IR and ISI (estimates of insulin sensitivity) were worse in male descendents from fructose-fed mothers in comparison to the other two groups, and these findings were also accompanied by a higher leptinemia. Interestingly, plasma AOPP and uricemia (oxidative stress markers) were augmented in male rats from fructose-fed mothers compared to the animals from control or glucose-fed mothers. In contrast, female rats did not show any differences in leptinemia between the three groups. Further, insulin sensitivity was significantly improved in fasted female rats from carbohydrate-fed mothers. In addition, plasma AOPP levels tended to be diminished in female rats from carbohydrate-fed mothers. Conclusion. Maternal fructose intake induces insulin resistance, hyperleptinemia, and plasma oxidative stress in male, but not female, progeny.

\section{Introduction}

In the last few decades, obesity, metabolic syndrome, and diabetes have escalated to epidemic proportions in many countries worldwide. These metabolic diseases are multifactorial resulting from genetic, physiological, behavioural, and environmental influences. Genetic influence alone does not suffice to explain the rate at which these diseases have increased [1]. In fact, several studies demonstrate that metabolic events during pre- and postnatal development modulate metabolic disease risks in later life [2]. Among them, feeding conditions likely constitute one of the most influential parameters on the health of the adult [3]. Thus, diet manipulation in mothers during critical developmental periods (such as gestation and/or the early postnatal) has been used to identify their contribution to obesity and diabetes development in offspring [4]. Given the current worldwide shift toward a westernized lifestyle, there is an urgent need to address the relationship between the quality and quantity of nutrient intake during pregnancy and/or lactation and the metabolic fate of the offspring [5].

Fructose, present in added sugars such as sucrose and high-fructose corn syrup, has been linked to obesity and metabolic syndrome [6-8]. Experimental studies have shown that fructose can induce leptin resistance and features of metabolic syndrome in rats, whereas glucose intake does not [9-11]. Clinical studies also support fructose as a cause of metabolic syndrome [12-14].

Interestingly, sex-dependent differences in the influence of fructose for inducing metabolic diseases have been reported. Thus, women, but not men, exhibit an association between fructose consumption and an increased risk of type 2 diabetes mellitus. In relation to this, it has been shown that female rats subjected to fructose have a more detrimental 
response than their male counterparts. Fructose-fed male rats were resistant to the hepatic effects of leptin, whereas fructose-fed females had no signs of leptin resistance but had hyperinsulinemia and altered glucose tolerance test [15]. In contrast, in high-fructose-fed rodents, oxidative stress was observed in male, but not in female, rats [16].

Since it has been well-established that fructose intake modifies lipidemia in laboratory animals and humans [11, 17] and maternal diet manipulations can affect the progeny, it might be speculated that fructose administration during gestation and/or lactation could cause metabolic changes in the offspring. Unfortunately, studies investigating altered maternal nutrition have used quite different experimental designs to determine the role of fructose [18-24].

In our previous report, we investigated the effects of a low fructose intake throughout gestation in mothers and their fetuses [25], and we obtained intriguing results. Fructosefed mothers presented a diminished leptin response to fasting and refeeding, their fetuses displaying an impaired transduction of the leptin signal, and these findings were not observed in glucose-fed rats. Therefore, the present study was designed to determine whether an intake of liquid fructose (10\% wt/vol) during pregnancy had long-term consequences on the offspring. Furthermore, special attention has been given to determine whether any potential consequence of the treatment differed between female and male offspring.

\section{Material and Methods}

2.1. Animals and Experimental Design. Female SpragueDawley rats weighing 200-240g were fed ad libitum a standard rat chow diet (B\&K Universal, Barcelona, Spain) and housed under controlled light and temperature conditions (12 h light-dark cycle; $22 \pm 1^{\circ} \mathrm{C}$ ). The experimental protocol was approved by the Animal Research Committee of the University CEU San Pablo, Madrid, Spain. The experimental protocol to which pregnant rats were subjected was the same as previously reported [25]. Briefly, pregnant animals were randomly separated into a control group, a fructosesupplemented group (fructose), and a glucose-supplemented group (glucose). Fructose and glucose were supplied as a $10 \%$ (wt/vol) solution in drinking water throughout gestation. Control animals received no supplementary sugar. Pregnant rats were allowed to deliver and, on the day of birth, each suckling litter was reduced to nine pups per mother. After delivery, both mothers and their pups were maintained with water and food ad libitum. It is remarkable that these animals (mothers and pups) received no subsequent additive in the drinking water. On the 21st day after delivery, the lactating mothers were removed to stop the suckling period and pups were separated by gender. When the progeny were 90 days old, they were subjected to 24-hour fasting. After drawing a basal blood sample from the tail vein, pellets were removed from the cages. After $24 \mathrm{~h}$ fasting, a second blood sample from the tail vein was obtained. Blood samples were collected into EDTA $(1 \mathrm{mg} / \mathrm{mL})$ tubes and placed on ice. Samples were then centrifuged, and plasma was stored at $-80^{\circ} \mathrm{C}$ until processed for glucose, insulin, leptin, and other determinations. Where two or three pups from one litter were used, their data were averaged.

2.2. Determinations. Plasma aliquots were used to measure glucose by an enzymatic colorimetric test (Spinreact, Girona, Spain). NEFA (nonesterified ("free") fatty acids) (Wako, Neuss, Germany), glycerol (Sigma Chemical, St. Louis, MO), triglycerides, and uric acid (Spinreact) were measured using commercial kits. Ketone bodies were measured using a kinetic method (Randox Laboratories, United Kingdom). Insulin was determined in plasma samples using a specific ELISA kit for rats (Mercodia, Uppsala, Sweden). Leptin and adiponectin were assayed in plasma samples using a specific enzyme immunoassay (EIA) kit for rats (Biovendor, Brno, Czech Republic, and Millipore, Bedford, MA, resp.).

Estimates of insulin resistance were calculated as previously described $[15,25]$ by determination of the following indexes from the $24 \mathrm{~h}$ fasting plasma glucose and insulin values: homeostasis model assessment of insulin resistance (HOMA-IR) and insulin sensitivity index (ISI). The HOMAIR was calculated as the product of the fasting plasma glucose (FPG) and insulin (FPI) divided by a constant, 22.5. FPI was expressed in microunits per milliliter and FPG as millimoles per liter [26]. The ISI was calculated as the ratio $2 /[(\mathrm{FPI} \times \mathrm{FPG})+1]$, expressing FPI and FPG in micromoles per liter [15]. Additionally, estimates of insulin sensitivity were also calculated in fed-state as previously described [25] by determination of the ratio between glucose levels (in milligrams per deciliter) and insulin values (in microunits per milliliter).

Finally, plasma aliquots were also used to determine the oxidative stress state. The concentration of malondialdehyde (MDA) in plasma was measured as a marker of lipid peroxidation using the method previously described [27], by measuring the fluorescence of MDA-thiobarbituric acid (TBA) complexes at $515 \mathrm{~nm} / 553 \mathrm{~nm}$ excitation/emission wavelengths. Further, the advanced oxidation protein products (AOPP) in plasma were determined as a protein oxidative stress biomarker using the spectrophotometric technique previously described $[28,29]$. The AOPP concentrations were expressed as $\mu \mathrm{mol} / \mathrm{L}$ of chloramine-T equivalents.

2.3. Statistical Analysis. Results were expressed as mean $\pm \mathrm{SE}$. Treatment effects were analyzed by one-way ANOVA. When treatment effects were significantly different $(P<0.05)$, means were tested by the Tukey multiple range test, using a computer program SSPS (version 15). When the variance was not homogeneous, a post hoc Tamhane test was performed.

\section{Results}

3.1. Ingestion of a $10 \% \mathrm{wt} / \mathrm{vol}$ Fructose Solution throughout Gestation Affects Leptinemia in Male Progeny. As shown in Table 1, neither fructose nor glucose intake throughout pregnancy produced alterations in the body weight of the male and female progeny. 
TABLE 1: Body weight and plasma analytes in fed 90-day-old progeny from fructose- or glucose-supplemented mothers.

\begin{tabular}{lccc}
\hline & \multicolumn{3}{c}{ Male } \\
& Control & Fructose & Glucose \\
\hline Body weight $(\mathrm{g})$ & $423.7 \pm 12.3$ & $403.4 \pm 12.8$ & $384.7 \pm 21.3$ \\
Glucose $(\mathrm{mg} / \mathrm{dL})$ & $133.4 \pm 4.3$ & $128.7 \pm 3.2$ & $138.0 \pm 1.0$ \\
Insulin $(\mu \mathrm{g} / \mathrm{L})$ & $0.53 \pm 0.05$ & $0.62 \pm 0.06$ & $0.47 \pm 0.08$ \\
Glucose/insulin ratio & $10.4 \pm 0.4$ & $8.1 \pm 0.9$ & $11.9 \pm 1.4$ \\
Triglycerides $(\mathrm{mg} / \mathrm{dL})$ & $95.6 \pm 8.0$ & $99.9 \pm 2.2$ & $82.5 \pm 5.7$ \\
NEFA $(\mathrm{mM})$ & $0.45 \pm 0.10$ & $0.56 \pm 0.15$ & $0.46 \pm 0.07$ \\
Glycerol $(\mathrm{mg} / \mathrm{dL})$ & $2.87 \pm 0.29$ & $3.18 \pm 0.22$ & $2.55 \pm 0.18$ \\
Adiponectin $(\mu \mathrm{g} / \mathrm{mL})$ & $18.9 \pm 1.1^{\mathrm{a}}$ & $24.3 \pm 0.9^{\mathrm{b}}$ & $25.1 \pm 1.8^{\mathrm{b}}$ \\
Leptin $(\mathrm{ng} / \mathrm{mL})$ & $6.35 \pm 0.57^{\mathrm{a}}$ & $11.20 \pm 1.44^{\mathrm{b}}$ & $7.08 \pm 0.92^{\mathrm{a}}$ \\
\hline & & Female & \\
& Control & Fructose & Glucose \\
\hline Body weight $(\mathrm{g})$ & $260.4 \pm 9.6$ & $258.7 \pm 6.4$ & $253.7 \pm 4.2$ \\
Glucose $(\mathrm{mg} / \mathrm{dL})$ & $139.3 \pm 7.0$ & $134.1 \pm 2.8$ & $142.7 \pm 1.8$ \\
Insulin $(\mu \mathrm{g} / \mathrm{L})$ & $0.54 \pm 0.05$ & $0.49 \pm 0.07$ & $0.40 \pm 0.05$ \\
Glucose/insulin ratio & $10.7 \pm 1.9$ & $10.0 \pm 0.7$ & $14.7 \pm 1.0$ \\
Triglycerides $(\mathrm{mg} / \mathrm{dL})$ & $57.2 \pm 2.9^{\mathrm{a}}$ & $55.9 \pm 8.1^{\mathrm{a}}$ & $32.0 \pm 4.3^{\mathrm{b}}$ \\
NEFA $(\mathrm{mM})$ & $0.48 \pm 0.09$ & $0.50 \pm 0.09$ & $0.37 \pm 0.04$ \\
Glycerol $(\mathrm{mg} / \mathrm{dL})$ & $2.81 \pm 0.21$ & $2.68 \pm 0.29$ & $2.10 \pm 0.14$ \\
Adiponectin $(\mu \mathrm{g} / \mathrm{mL})$ & $43.9 \pm 4.0$ & $48.7 \pm 3.5$ & $46.7 \pm 3.8$ \\
Leptin $(\mathrm{ng} / \mathrm{mL})$ & $5.27 \pm 0.50$ & $4.61 \pm 0.24$ & $4.41 \pm 0.17$ \\
\hline & & & \\
& & &
\end{tabular}

Data are means \pm SE; $n=10-12$ animals from four litters. Where two or three pups from one litter were studied, their data were averaged. Different letters indicate significant differences between the groups $(P<0.05)$.

Plasma NEFA, glycerol, and triglycerides concentrations were similar in the male rats from carbohydratefed mothers with respect to control values (Table 1). Male rats from carbohydrate-fed mothers showed higher levels of plasma adiponectin levels. Although glycemia and insulinemia showed no differences between the three groups (Table 1), glucose/insulin ratio tended to be lower in the male animals from fructose-fed mothers compared to the animals from control and glucose-supplemented rats (Table 1). Interestingly, male descendents from fructose-supplemented rats turned out to be clearly hyperleptinemic (Table 1).

On the other hand, since insulinemia and glycemia were similar in the female rats from carbohydrate-fed mothers with respect to control values, glucose/insulin ratio was found to be similar for the female progeny of control, fructosefed, and glucose-fed pregnant rats (Table 1). Similar findings were recorded for plasma adiponectin levels. However, plasma NEFA, glycerol, and triglycerides concentrations were lower in female animals from glucose-fed pregnant rats in comparison to the other two groups (Table 1). In contrast to males, female descendents from fructose-supplemented rats showed similar values in their leptinemia to the animals from control and glucose-supplemented mothers (Table 1).

3.2. Ingestion of a $10 \% \mathrm{wt} / \mathrm{vol}$ Fructose Solution throughout Gestation Affects Insulin Sensitivity in Fasted Male Progeny. To confirm a possible disturbance in glucose homeostasis in
TABLE 2: Plasma analytes in fasted 91-day-old progeny from fructose- or glucose-supplemented mothers.

\begin{tabular}{lccc}
\hline & \multicolumn{3}{c}{ Male } \\
& Control & Fructose & Glucose \\
\hline Glucose $(\mathrm{mg} / \mathrm{dL})$ & $89.3 \pm 6.1$ & $97.6 \pm 4.8$ & $96.8 \pm 5.7$ \\
Insulin $(\mu \mathrm{g} / \mathrm{L})$ & $0.044 \pm 0.015^{\mathrm{a}}$ & $0.144 \pm 0.031^{\mathrm{b}}$ & $0.031 \pm 0.006^{\mathrm{a}}$ \\
Triglycerides $(\mathrm{mg} / \mathrm{dL})$ & $52.1 \pm 12.6$ & $53.8 \pm 6.8$ & $35.6 \pm 4.1$ \\
NEFA $(\mathrm{mM})$ & $1.66 \pm 0.24$ & $1.37 \pm 0.13$ & $1.42 \pm 0.06$ \\
Glycerol $(\mathrm{mg} / \mathrm{dL})$ & $5.04 \pm 0.72$ & $4.12 \pm 0.34$ & $4.25 \pm 0.15$ \\
Adiponectin $(\mu \mathrm{g} / \mathrm{mL})$ & $29.9 \pm 6.8$ & $27.7 \pm 3.6$ & $21.4 \pm 1.0$ \\
Ketone bodies $(\mathrm{mM})$ & $0.64 \pm 0.16$ & $0.63 \pm 0.11$ & $0.73 \pm 0.06$ \\
\hline & & Female \\
& Control & Fructose & Glucose \\
\hline Glucose $(\mathrm{mg} / \mathrm{dL})$ & $104.1 \pm 6.6$ & $94.8 \pm 2.8$ & $102.9 \pm 7.4$ \\
Insulin $(\mu \mathrm{g} / \mathrm{L})$ & $0.101 \pm 0.017^{\mathrm{a}}$ & $0.042 \pm 0.010^{\mathrm{b}}$ & $0.026 \pm 0.004^{\mathrm{b}}$ \\
Triglycerides $(\mathrm{mg} / \mathrm{dL})$ & $26.4 \pm 8.9$ & $24.7 \pm 4.4$ & $23.6 \pm 4.6$ \\
NEFA $(\mathrm{mM})$ & $1.49 \pm 0.29$ & $1.49 \pm 0.21$ & $1.29 \pm 0.04$ \\
Glycerol $(\mathrm{mg} / \mathrm{dL})$ & $4.62 \pm 0.43$ & $4.32 \pm 0.48$ & $4.94 \pm 0.58$ \\
Adiponectin $(\mu \mathrm{g} / \mathrm{mL})$ & $47.8 \pm 8.6$ & $48.2 \pm 2.0$ & $37.6 \pm 5.3$ \\
Ketone bodies $(\mathrm{mM})$ & $0.63 \pm 0.10$ & $0.97 \pm 0.12$ & $1.00 \pm 0.20$ \\
\hline
\end{tabular}

Data are means \pm SE; $n=10-12$ animals from four litters. Where two or three pups from one litter were studied, their data were averaged. Different letters indicate significant differences between the groups $(P<0.05)$.

male, but not in female, rats born of mothers supplemented throughout pregnancy with liquid fructose, we subjected the progeny of the three experimental groups to $24 \mathrm{~h}$ fasting and measured plasma parameters related to insulin resistance.

After 24-hour fasting, plasma glucose, NEFA, glycerol, ketone bodies, and triglycerides concentrations did not show any differences in the male rats from carbohydrate-fed mothers with respect to control values (Table 2). Similar findings were recorded for plasma adiponectin levels. However, insulinemia was significantly higher in male animals from fructose-fed pregnant rats in comparison to the other two groups (Table 2). Thus, HOMA-IR and ISI ratios were significantly different in the male animals from fructose-fed mothers compared to the male animals from control and glucose-supplemented rats (Figures 1(a)-1(b)), and, therefore, these findings confirmed that an insulin resistant state exists in male progeny of fructose-fed mothers. In accordance with this result, male descendents from fructose-supplemented rats showed a clear increase in their plasma leptin concentrations (Figure 1(c)).

After 24-hour fasting, plasma glucose, NEFA, glycerol, ketone bodies, adiponectin, and triglycerides levels were similar in the female rats from carbohydrate-fed mothers with respect to the control values (Table 2 ). However, insulinemia was lower in female animals from carbohydrate-fed pregnant rats in comparison to the control group (Table 2). Thus, HOMA-IR and ISI ratios were significantly different in the female animals from carbohydrate-supplemented mothers compared to the female animals from control rats (Figures 1(a)-1(b)), these being more pronounced in the group from the glucose-fed mothers. Thus, not only did female rats from fructose-fed mothers not show an insulin 

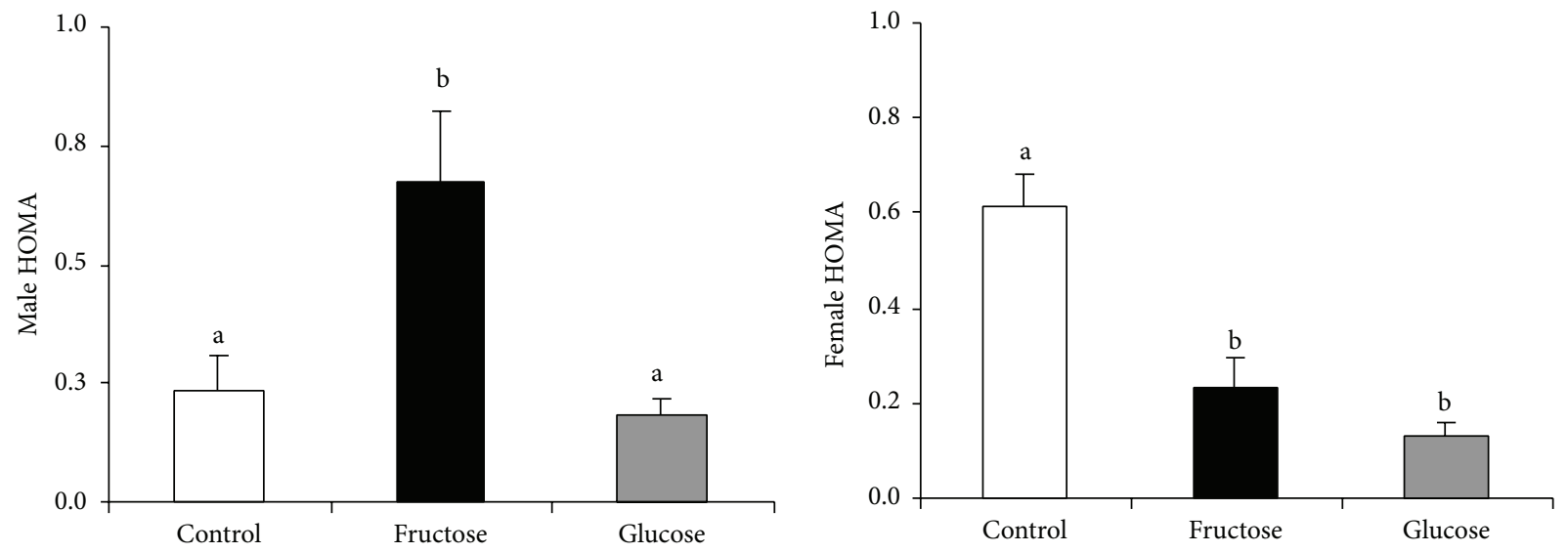

(a)
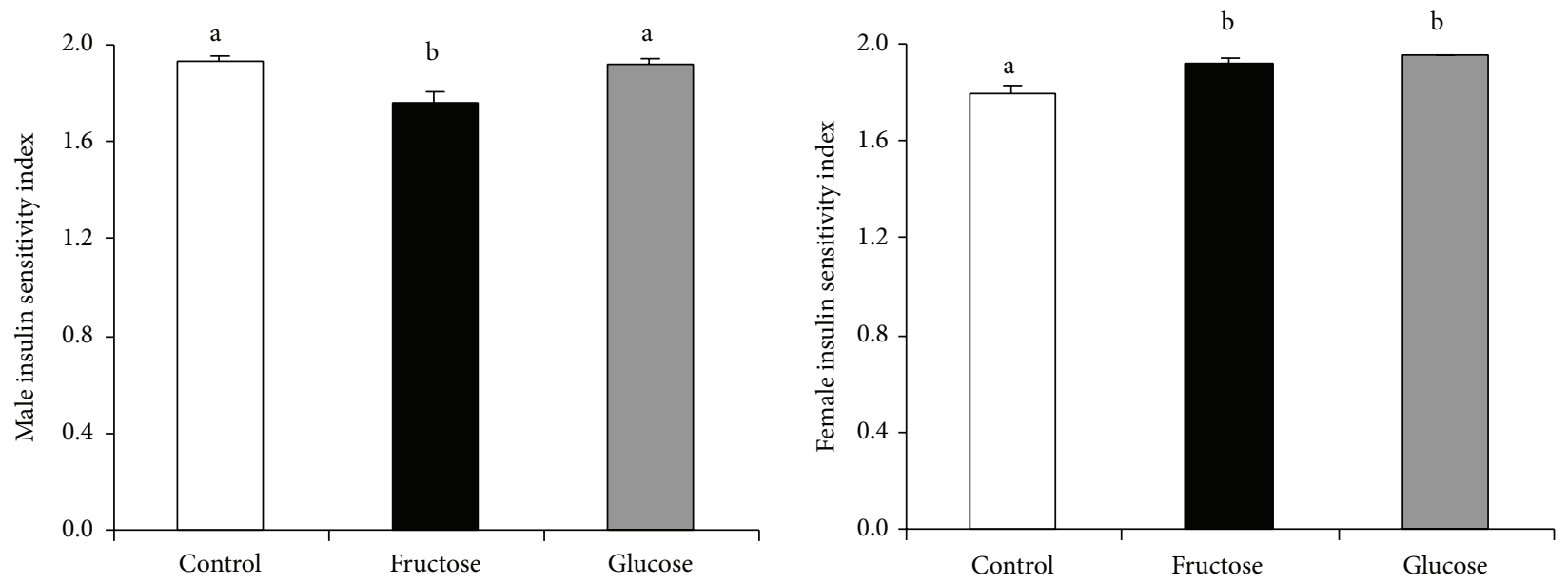

(b)
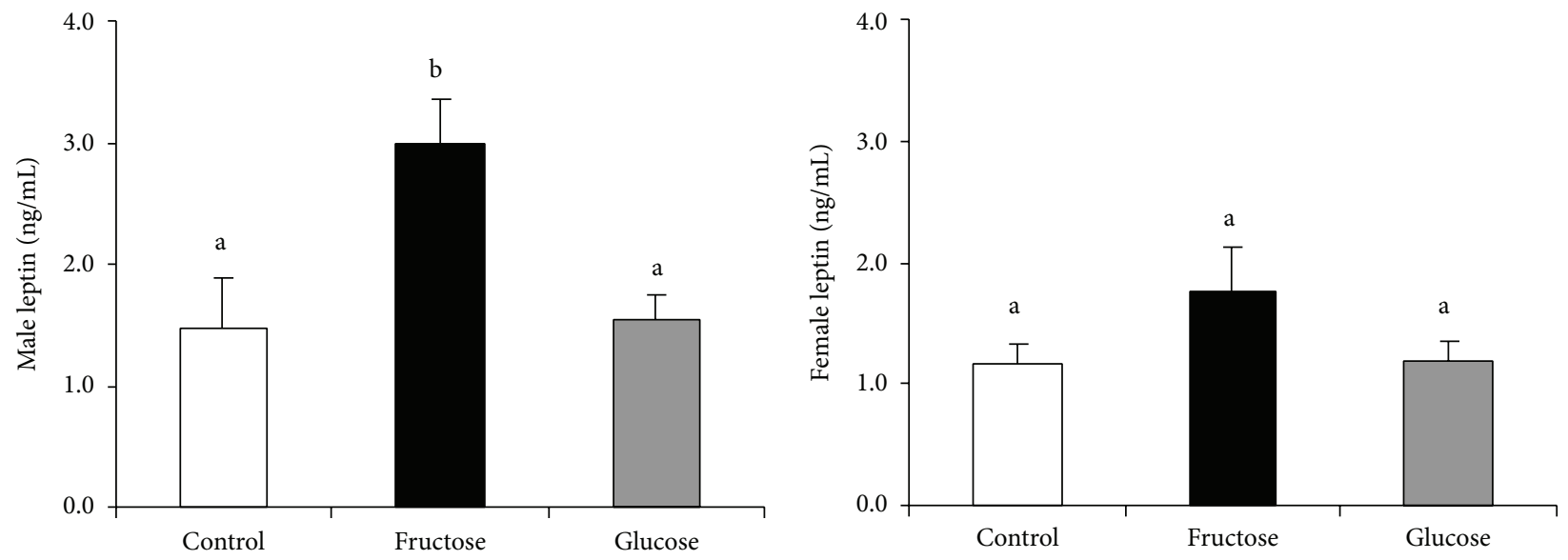

(c)

FIGURE 1: Fructose in pregnancy produces insulin resistance and hyperleptinemia in progeny. Male and female (a) HOMA-IR and (b) ISI ratios and (c) leptinemia of 24-hour fasted 90-day-old progeny from control, fructose-fed, and glucose-fed pregnant rats. Data are means \pm SE; $n=10-12$ animals from four litters. Different letters indicate significant differences between the groups $(P<0.05)$. 

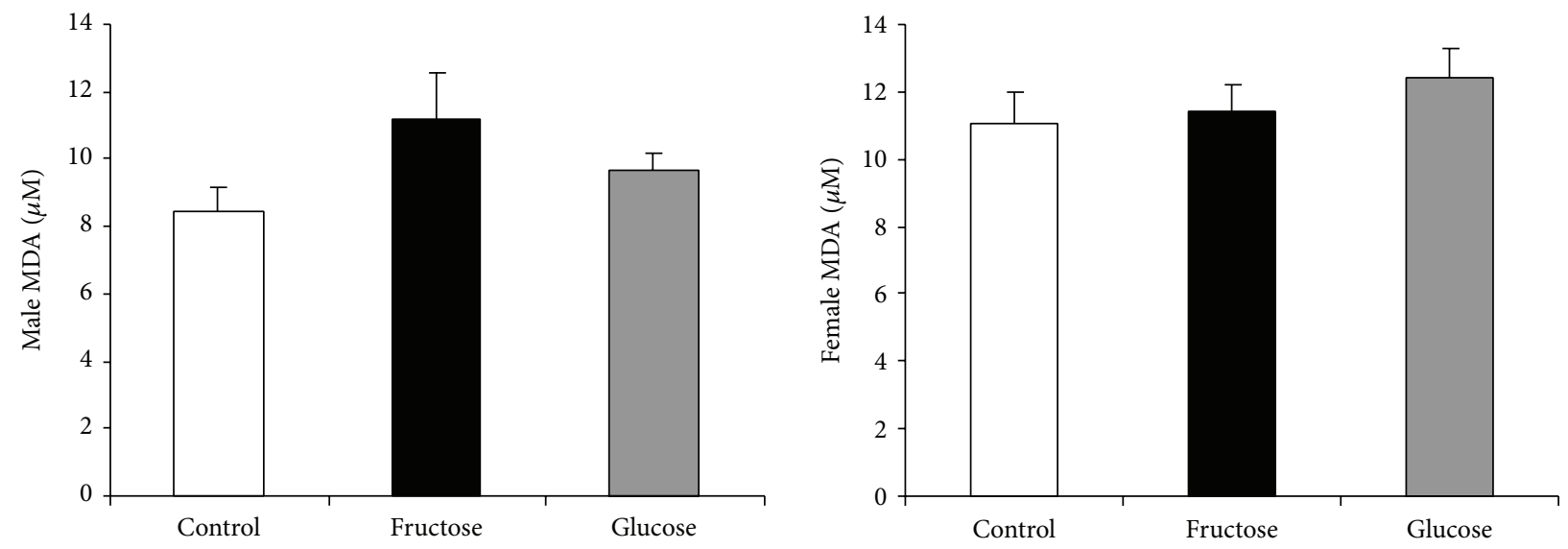

(a)
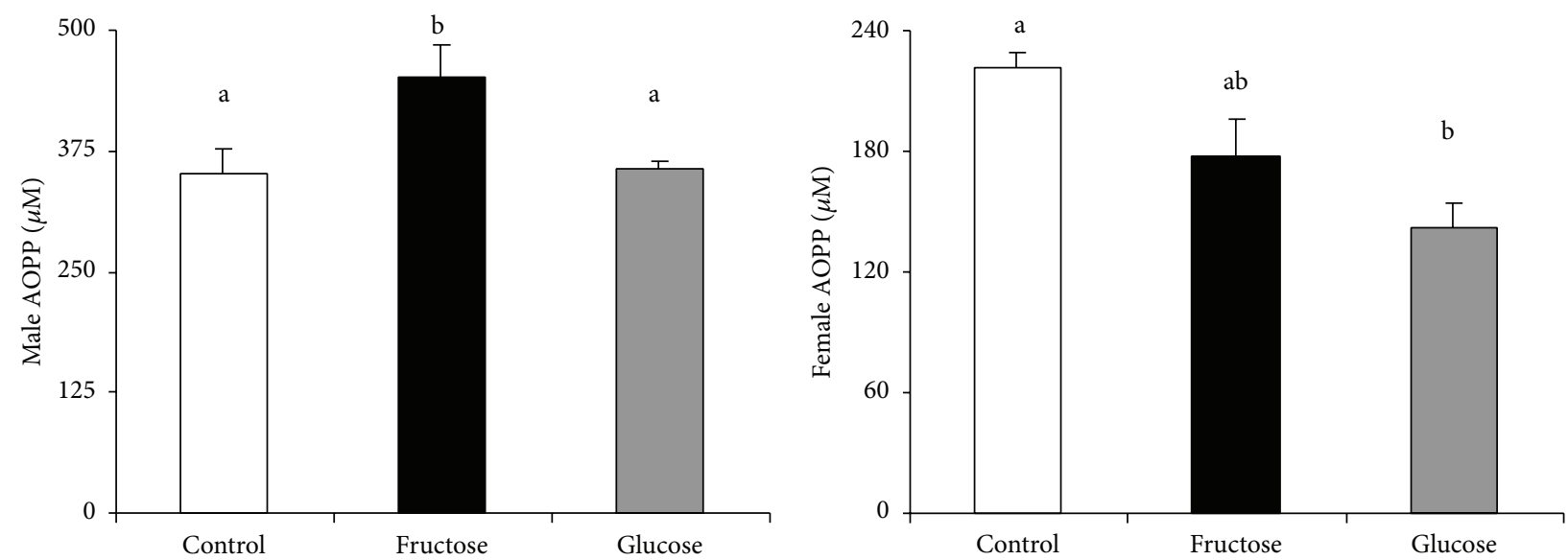

(b)

FIGURE 2: Fructose in pregnancy influences plasma oxidative stress in progeny. Male and female (a) plasma MDA (b) and AOPP values of 90-day-old progeny from control, fructose-fed, and glucose-fed pregnant rats. Data are means \pm SE; $n=10-12$ animals from four litters. Different letters indicate significant differences between the groups $(P<0.05)$.

resistant state as seen in male progeny, but female rats from carbohydrate-fed mothers showed improved insulin sensitivity. In fasted female descendents from fructose-supplemented rats, although plasma leptin concentrations tended to be higher than in the other two groups, the effect was not significant (Figure 1(c)).

\subsection{Ingestion of a $10 \% \mathrm{wt} / \mathrm{vol}$ Fructose Solution throughout} Gestation Affects Plasma Oxidative Stress in Male Progeny. Since fructose intake during pregnancy seems to produce insulin resistance and hyperleptinemia in the male progeny, parameters which have been related to metabolic syndrome, we also checked whether other features related to that disturbance could be affected. Thus, as shown in Figure 2, plasma MDA and AOPP, which would indicate lipid and protein oxidation, respectively, tended to be augmented in the male rats from fructose-fed mothers in comparison to the animals from control and glucose-fed pregnant rats, becoming significant for AOPP levels. In contrast, plasma AOPP, but not MDA, were diminished in the female progeny of carbohydrate-fed mothers (Figure 2), this effect being significantly different in the progeny from glucose-fed mothers.

Interestingly, male rats from fructose-supplemented mothers showed higher uricemia $(3.4 \pm 0.5 ; 5.4 \pm 0.3$; and $3.4 \pm 0.4 \mathrm{mg} / \mathrm{dL}$, for males born from control, fructose-fed, and glucose-fed mothers, resp., $P<0.05)$. In comparison, females from carbohydrate-fed mothers showed lower levels of plasma uric acid than the control group, this effect being significantly different in the progeny from glucose-fed mothers $(4.1 \pm 0.3 ; 3.4 \pm 0.3$; and $2.3 \pm 0.2 \mathrm{mg} / \mathrm{dL}$, for female progeny from control, fructose-fed, and glucose-fed mothers, resp., $P<0.05)$.

\section{Discussion}

In our previous study [25], we reported an impaired hepatic transduction of the leptin signal in the fetuses from fructosefed, but not glucose-fed, pregnant rats and related it to a diminished leptin response to fasting and refeeding observed in their mothers. Since it has been proposed that a period of relative hypoleptinemia (or, in our case, leptin resistance) 
during development may induce some metabolic adaptations that underlie developmental programming [30], we speculated whether the findings found in our previous study [25] could be responsible for a developmental programming of those progeny and produce some metabolic disturbances, when adult.

Thus, male progeny born of fructose-fed rats presented higher leptinemia versus the other two groups, and that hyperleptinemia was observed in both fed and fasting conditions. In accordance with this finding, fasting insulinemia was higher in the male progeny from fructose-fed mothers, all these results being consistent with the insulin resistant state found in these animals (measured as HOMA and ISI ratios). The presence of hyperinsulinemia in fasted male fructosefed progeny along with hyperleptinemia could also indicate leptin resistance at the level of pancreatic islets [31]. Since insulin stimulates adipogenesis and leptin production in adipocytes whereas leptin inhibits the production of insulin in pancreatic $\beta$-cells, a prolonged elevation of plasma leptin levels would result in dysregulation of the adipoinsular axis and a corresponding failure to suppress insulin secretion [32]. In fact, in the present study, although the male progeny from fructose-fed mothers was hyperleptinemic in both fed and fasted conditions, fasting produced a lower impact on insulin levels in comparison to the other two groups (12.1-, 4.3-, and 15.2-fold reduction, for control, fructose, and glucose groups, resp.). In relation to this, it has been reported that adipocytes from male offspring of lactating mothers consuming fructose spontaneously released more leptin than control rat-derived adipocytes and also displayed impaired response to insulin stimulation [4]. Since the progeny from fructose-fed mothers were already leptin resistant when they were fetuses [25], the male progeny of fructose-fed pregnant rats could present a vicious circle (leptin resistance, hypersecretion of insulin, and increasing insulin resistance) $[23,33]$.

On the other hand, it has been demonstrated that fructose is much more reactive than glucose with respect to participation in glycosylation reactions, which represent an important source of free radicals. In accordance with this fact, rats fed fructose (25\% wt/vol) exhibited a significant increase in lipid peroxidation products, compared with rats fed the same amount of glucose or sucrose and those given pure water [34]. Moreover, it has been reported that a short-term administration of a fructose-rich diet to normal rats promotes an increase in several glycoxidative stress markers [16, 35]. Further, as a reducing sugar, fructose reacts with protein molecules to form toxic advanced glycation end-products (AGEs) and also induces protein oxidation possibly through the formation of hydroxyl radicals [36]. Both AGEs and AOPP appear to be involved in the pathogenesis of inflammation, diabetes complications, and cardiovascular diseases. In the present work, the male progeny from fructose-fed dams showed increased plasma levels of protein oxidation products, measured as AOPP. It is worthwhile to mention that AOPP levels have been associated with metabolic syndrome, since high levels of AOPP have been positively correlated with insulin and HOMA levels [37]. Given that it has been proposed that AGEs could accumulate indefinitely on longlived molecules such as collagen and DNA [38], it might be speculated that fructose intake during pregnancy could promote oxidation products formation in fetuses and these toxic compounds would accumulate, later appearing in the plasma of progeny.

Related to that increase in plasma oxidative stress of progeny from fructose-fed rats, we also determined uricemia and, surprisingly, male rats born to fructose-fed mothers turned out to be hyperuricemic. Some authors consider hyperuricemia in metabolic syndrome to be the consequence of elevated serum insulin levels, which have been shown to stimulate renal reabsorption of uric acid. In fact, thiazolidinediones which improve insulin sensitivity and lower insulin levels reduce the level of serum uric acid in diabetic patients. Conversely, Nakagawa et al. (2006) demonstrated that the reduction of uric acid levels with a xanthine oxidase inhibitor improved insulin sensitivity. Accordingly, in the present study, fructose-fed male progeny presented hyperuricemia along with insulin resistance. Interestingly, the xanthine oxidase pathway, which is related to uric acid production, has been shown to generate oxidants [39]. Our findings would agree with this result since the hyperuricemic male progeny also presents an increase in plasma oxidative stress. In fact, a recent study has shown a close relationship between fructose, hyperuricemia, oxidative stress, and diabetes [40].

The most prominent result found here is that the intake of just a small amount of fructose (10\%) throughout gestation produces a clear impairment in the insulin action, hyperleptinemia, and other features of metabolic syndrome such as high values of oxidative stress biomarkers and uricemia in male progeny. However, female progeny born of fructose-fed mothers did not show any of these characteristics of metabolic syndrome. Nevertheless, as has been described, females born to mothers subjected to undernutrition expressed a programmed phenotype only in the presence of a high-fat diet, whereas the male progeny could manifest it independently of postnatal nutrition [30]. Therefore, it is possible that postnatal hypercaloric nutrition could amplify all these metabolic abnormalities induced by the fructose-fed fetal programming, and this deserves further investigation.

Finally, since the carbohydrate was administered only during gestation, we can assume that the effects of fructose intake would occur during intrauterine development. As previously reported [25], fetuses from fructose-fed mothers presented leptin resistance. Leptin has been implicated in the regulation of insulin secretion by islets and, in fact, leptin may influence the normal proliferation of pancreatic $\beta$-cells which occurs in the neonatal period [30]. If it is assumed that an interrelated endocrine insulin-leptin feedback system exists, we could speculate that the adipoinsular axis has been affected by fructose intake throughout pregnancy.

\section{Conclusion}

Maternal fructose intake seems to provoke features of metabolic syndrome, namely, insulin resistance, hyperleptinemia, hyperuricemia, and plasma oxidative stress in male, but not female, progeny. 


\section{Conflict of Interests}

The authors declare that there is no conflict of interests regarding the publication of this paper.

\section{Acknowledgments}

The authors thank Jose M. Garrido for his help in handling the rats and Brian Crilly for his editorial help. This work was supported by a grant from Plan Nacional de Investigación Científica, Desarrollo e Innovación Tecnológica (I+D+i), Instituto de Salud Carlos III-Subdirección General de Evaluación y Fomento de la Investigación (PI-09/02192), European Community FEDER, and Fundación Universitaria San Pablo CEU (PC 09/2012). Silvia Rodrigo is a FUSP-CEU fellowship.

\section{References}

[1] G. J. Howie, D. M. Sloboda, T. Kamal, and M. H. Vickers, "Maternal nutritional history predicts obesity in adult offspring independent of postnatal diet," The Journal of Physiology, vol. 587, no. 4, pp. 905-915, 2009.

[2] B. Koletzko, I. Broekaert, H. Demmelmair et al., "Protein intake in the first year of life: a risk factor for later obesity? The E.U. childhood obesity project," Advances in Experimental Medicine and Biology, vol. 569, pp. 69-79, 2005.

[3] B. Beck, S. Richy, Z. A. Archer, and J. G. Mercer, "Ingestion of carbohydrate-rich supplements during gestation programs insulin and leptin resistance but not body weight gain in adult rat offspring," Frontiers in Physiology, vol. 3, article 224, 2012.

[4] A. Alzamendi, D. Castrogiovanni, R. C. Gaillard, E. Spinedi, and A. Giovambattista, "Increased male offspring's risk of metabolic-neuroendocrine dysfunction and overweight after fructose-rich diet intake by the lactating mother," Endocrinology, vol. 151, no. 9, pp. 4214-4223, 2010.

[5] L. Šedová, O. Šeda, L. Kazdová et al., "Sucrose feeding during pregnancy and lactation elicits distinct metabolic response in offspring of an inbred genetic model of metabolic syndrome," The American Journal of Physiology-Endocrinology and Metabolism, vol. 292, no. 5, pp. E1318-E1324, 2007.

[6] P. J. Havel, "Dietary fructose: implications for dysregulation of energy homeostasis and lipid/carbohydrate metabolism," Nutrition Reviews, vol. 63, no. 5, pp. 133-137, 2005.

[7] L. Tappy and K.-A. Lê, "Metabolic effects of fructose and the worldwide increase in obesity," Physiological Reviews, vol. 90, no. 1, pp. 23-46, 2010.

[8] R. J. Johnson, M. S. Segal, Y. Sautin et al., "Potential role of sugar (fructose) in the epidemic of hypertension, obesity and the metabolic syndrome, diabetes, kidney disease, and cardiovascular disease," The American Journal of Clinical Nutrition, vol. 86, no. 4, pp. 899-906, 2007.

[9] R. J. Johnson, S. E. Perez-Pozo, Y. Y. Sautin et al., "Hypothesis: could excessive fructose intake and uric acid cause type 2 diabetes?" Endocrine Reviews, vol. 30, no. 1, pp. 96-116, 2009.

[10] C. H. Taghibiglou, A. Carpentier, S. C. van Iderstine et al., "Mechanisms of hepatic very low-density lipoprotein overproduction in insulin resistance," The Journal of Biological Chemistry, vol. 275, no. 12, pp. 8416-8425, 2000.
[11] N. Roglans, L. Vilà, M. Farré et al., "Impairment of hepatic STAT- 3 activation and reduction of PPAR $\alpha$ activity in fructosefed rats," Hepatology, vol. 45, no. 3, pp. 778-788, 2007.

[12] K. L. Stanhope, J. M. Schwarz, N. L. Keim et al., "Consuming fructose-sweetened, not glucose-sweetened, beverages increases visceral adiposity and lipids and decreases insulin sensitivity in overweight/obese humans," Journal of Clinical Investigation, vol. 119, no. 5, pp. 1322-1334, 2009.

[13] K. L. Stanhope, A. A. Bremer, V. Medici et al., "Consumption of fructose and high fructose corn syrup increase postprandial triglycerides, LDL-cholesterol, and apolipoprotein-B in young men and women," The Journal of Clinical Endocrinology and Metabolism, vol. 96, no. 10, pp. E1596-E1605, 2011.

[14] L. de Koning, V. S. Malik, M. D. Kellogg, E. B. Rimm, W. C. Willett, and F. B. Hu, "Sweetened beverage consumption, incident coronary heart disease, and biomarkers of risk in men," Circulation, vol. 125, no. 14, pp. 1735-1741, 2012.

[15] L. Vilà, N. Roglans, V. Perna et al., "Liver AMP/ATP ratio and fructokinase expression are related to gender differences in AMPK activity and glucose intolerance in rats ingesting liquid fructose," Journal of Nutritional Biochemistry, vol. 22, no. 8, pp. 741-751, 2011.

[16] J. Busserolles, A. Mazur, E. Gueux, E. Rock, and Y. Rayssiguier, "Metabolic syndrome in the rat: females are protected against the pro-oxidant effect of a high sucrose diet," Experimental Biology and Medicine, vol. 227, no. 9, pp. 837-842, 2002.

[17] K.-A. Lê, D. Faeh, R. Stettler et al., "A 4-wk high-fructose diet alters lipid metabolism without affecting insulin sensitivity or ectopic lipids in healthy humans," The American Journal of Clinical Nutrition, vol. 84, no. 6, pp. 1374-1379, 2006.

[18] S. A. Bayol, B. H. Simbi, J. A. Bertrand, and N. C. Stickland, "Offspring from mothers fed a 'junk food' diet in pregnancy and lactation exhibit exacerbated adiposity that is more pronounced in females," The Journal of Physiology, vol. 586, no. 13, pp. 32193230, 2008.

[19] A. Soria, A. Chicco, N. Mocchiutti et al., "A sucrose-rich diet affects triglyceride metabolism differently in pregnant and nonpregnant rats and has negative effects on fetal growth," Journal of Nutrition, vol. 126, no. 10, pp. 2481-2486, 1996.

[20] M. A. Munilla and E. Herrera, "Maternal hypertriglyceridemia during late pregnancy does not affect the increase in circulating triglycerides caused by the long-term consumption of a sucroserich diet by rats," Journal of Nutrition, vol. 130, no. 12, pp. 28832888, 2000.

[21] K.-L. C. Jen, C. Rochon, S. Zhong, and L. Whitcomb, "Fructose and sucrose feeding during pregnancy and lactation in rats changes maternal and pup fuel metabolism," Journal of Nutrition, vol. 121, no. 12, pp. 1999-2005, 1991.

[22] R. H. H. Ching, L. O. Y. Yeung, I. M. Y. Tse, W.-H. Sit, and E. T. S. Li, "Supplementation of bitter melon to rats fed a high-fructose diet during gestation and lactation ameliorates fructose-induced dyslipidemia and hepatic oxidative stress in male offspring," Journal of Nutrition, vol. 141, no. 9, pp. 16641672, 2011.

[23] S. Rawana, K. Clark, S. Zhong, A. Buison, S. Chackunkal, and K.-L. C. Jen, "Low dose fructose ingestion during gestation and lactation affects carbohydrate metabolism in rat dams and their offspring," Journal of Nutrition, vol. 123, no. 12, pp. 2158-2165, 1993. 
[24] M. H. Vickers, Z. E. Clayton, C. Yap, and D. M. Sloboda, "Maternal fructose intake during pregnancy and lactation alters placental growth and leads to sex-specific changes in fetal and neonatal endocrine function," Endocrinology, vol. 152, no. 4, pp. 1378-1387, 2011.

[25] L. Rodríguez, M. I. Panadero, N. Roglans et al., "Fructose during pregnancy affects maternal and fetal leptin signaling," The Journal of Nutritional Biochemistry, vol. 24, no. 10, pp. 17091716, 2013.

[26] R. Muniyappa, S. Lee, H. Chen, and M. J. Quon, "Current approaches for assessing insulin sensitivity and resistance in vivo: advantages, limitations, and appropriate usage," The American Journal of Physiology - Endocrinology and Metabolism, vol. 294, no. 1, pp. E15-E26, 2008.

[27] S. H. Y. Wong, J. A. Knight, S. M. Hopfer, O. Zaharia, C. N. Leach Jr., and F. W. Sunderman Jr., "Lipoperoxides in plasma as measured by liquid-chromatographic separation of malondialdehyde-thiobarbituric acid adduct," Clinical Chemistry, vol. 33, no. 2, part 1, pp. 214-220, 1987.

[28] V. Witko-Sarsat, M. Friedlander, T. N. Khoa et al., "Advanced oxidation protein products as novel mediators of inflammation and monocyte activation in chronic renal failure," Journal of Immunology, vol. 161, no. 5, pp. 2524-2532, 1998.

[29] B. Anderstam, B.-H. Ann-Christin, A. Valli, P. Stenvinkel, B. Lindholm, and M. E. Suliman, "Modification of the oxidative stress biomarker AOPP assay: application in uremic samples," Clinica Chimica Acta, vol. 393, no. 2, pp. 114-118, 2008.

[30] M. H. Vickers, P. D. Gluckman, A. H. Coveny et al., "Neonatal leptin treatment reverses developmental programming," Endocrinology, vol. 146, no. 10, pp. 4211-4216, 2005.

[31] M. Srinivasan, C. Dodds, H. Ghanim et al., "Maternal obesity and fetal programming: effects of a high-carbohydrate nutritional modification in the immediate postnatal life of female rats," The American Journal of Physiology-Endocrinology and Metabolism, vol. 295, no. 4, pp. E895-E903, 2008.

[32] M. H. Vickers, S. Reddy, B. A. Ikenasio, and B. H. Breier, "Dysregulation of the adipoinsular axis-a mechanism for the pathogenesis of hyperleptinemia and adipogenic diabetes induced by fetal programming," Journal of Endocrinology, vol. 170, no. 2, pp. 323-332, 2001.

[33] A. Shapiro, W. Mu, C. Roncal, K.-Y. Cheng, R. J. Johnson, and P. J. Scarpace, "Fructose-induced leptin resistance exacerbates weight gain in response to subsequent high-fat feeding," The American Journal of Physiology-Regulatory Integrative and Comparative Physiology, vol. 295, no. 5, pp. R1370-R1375, 2008.

[34] B. Levi and M. J. Werman, "Long-term fructose consumption accelerates glycation and several age-related variables in male rats," Journal of Nutrition, vol. 128, no. 9, pp. 1442-1449, 1998.

[35] M. C. Castro, M. L. Massa, H. del Zotto, J. J. Gagliardino, and F. Francini, "Rat liver uncoupling protein 2: changes induced by a fructose-rich diet," Life Sciences, vol. 89, no. 17-18, pp. 609-614, 2011.

[36] Y. Takagi, A. Kashiwagi, Y. Tanaka, T. Asahina, R. Kikkawa, and Y. Shigeta, "Significance of fructose-induced protein oxidation and formation of advanced glycation end product," Journal of Diabetes and Its Complications, vol. 9, no. 2, pp. 87-91, 1995.

[37] G. G. Korkmaz, E. Altinoglu, S. Civelek et al., "The association of oxidative stress markers with conventional risk factors in the metabolic syndrome," Metabolism: Clinical and Experimental, vol. 62 , no. 6 , pp. 828-835, 2013.
[38] A. R. Gaby, "Adverse effects of dietary fructose," Alternative Medicine Review, vol. 10, no. 4, pp. 294-306, 2005.

[39] T. Nakagawa, H. Hu, S. Zharikov et al., "A causal role for uric acid in fructose-induced metabolic syndrome," American Journal of Physiology-Renal Physiology, vol. 290, no. 3, pp. F625-F631, 2006.

[40] R. J. Johnson, T. Nakagawa, L. G. Sanchez-Lozada et al., "Sugar, uric acid, and the etiology of diabetes and obesity," Diabetes, vol. 62 , no. 10 , pp. 3307-3315, 2013. 


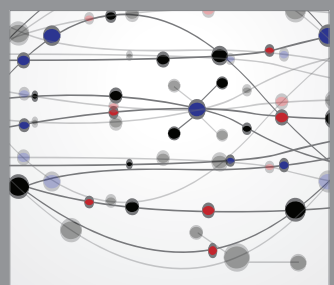

The Scientific World Journal
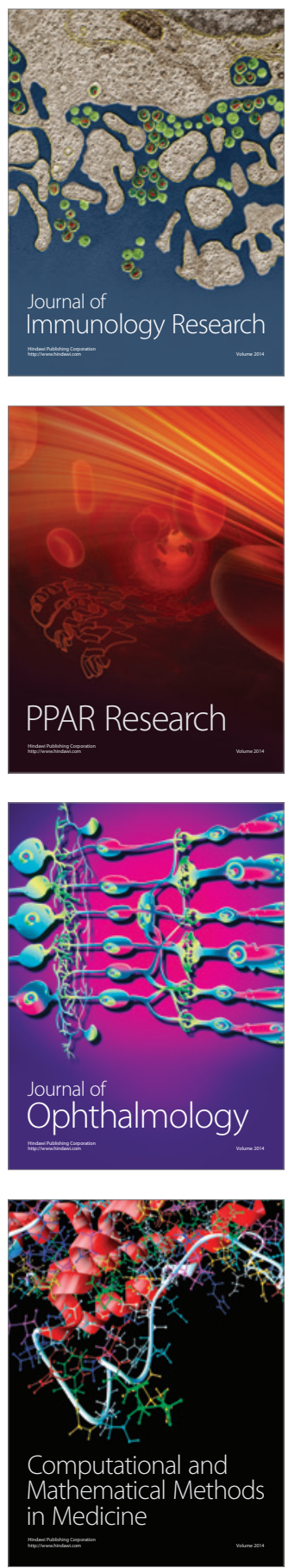

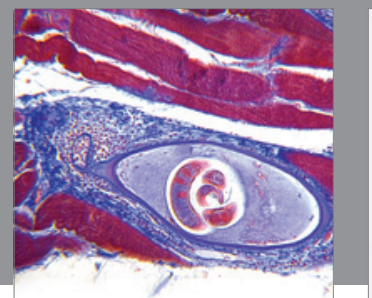

Gastroenterology

Research and Practice
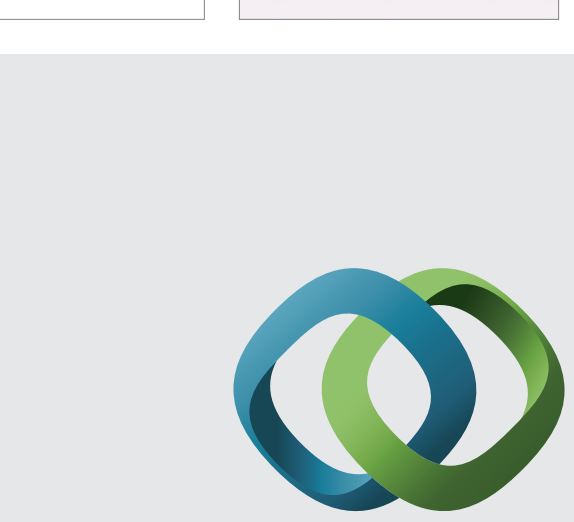

\section{Hindawi}

Submit your manuscripts at

http://www.hindawi.com
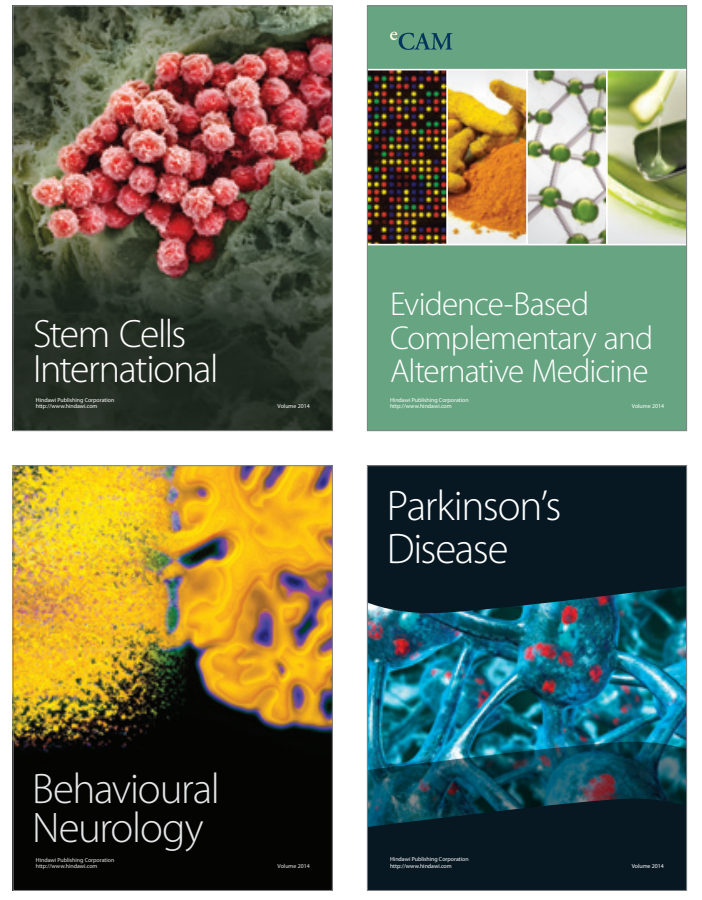
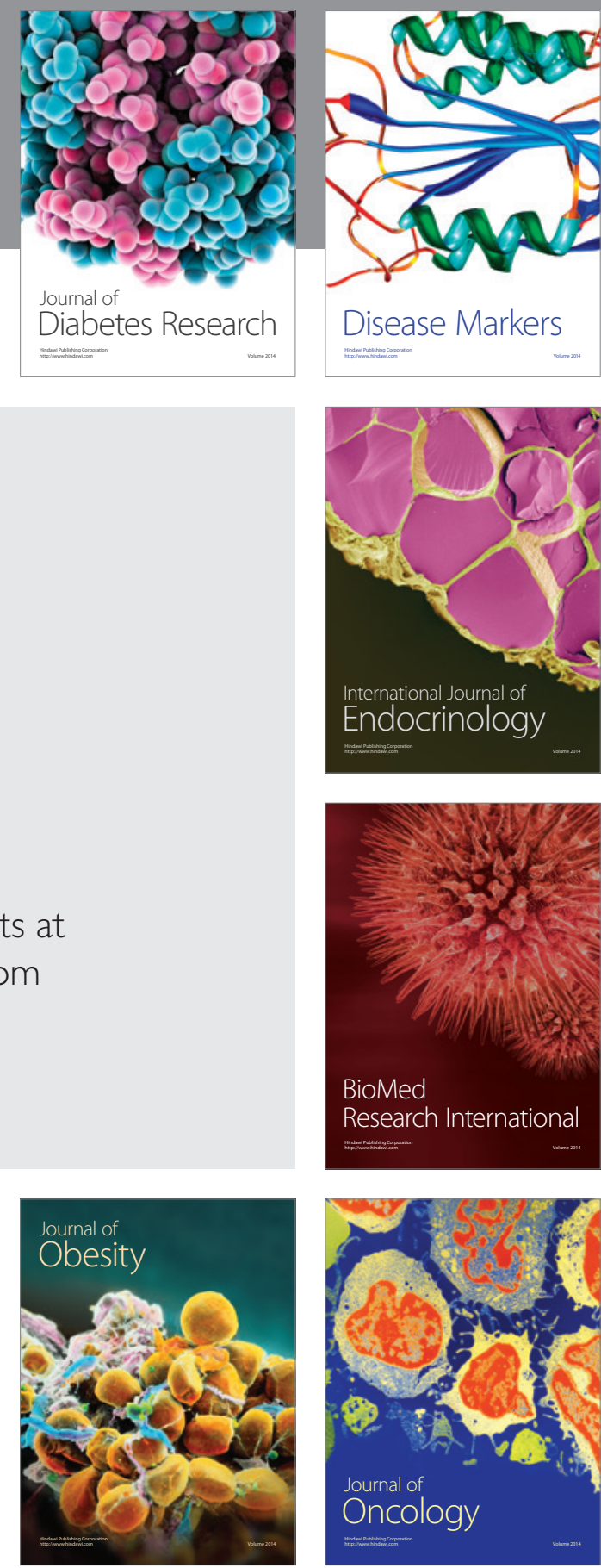

Disease Markers
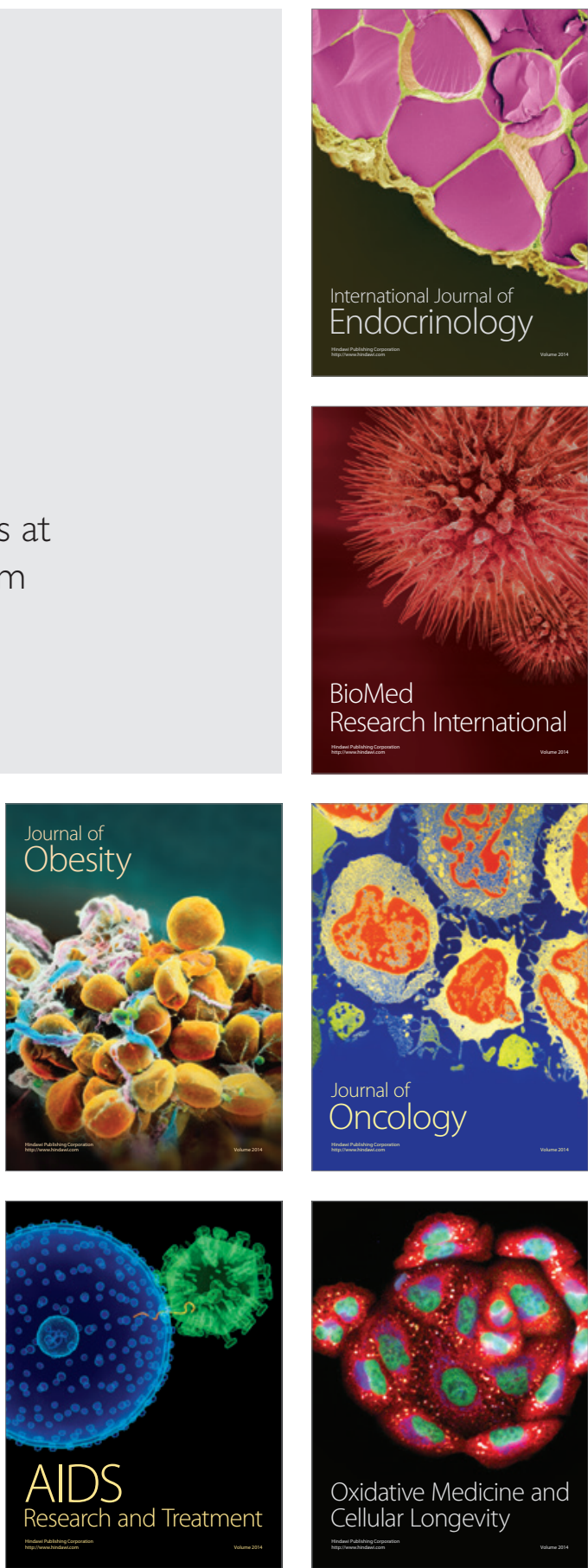